\title{
Performance Evaluation of GPSR and AODV Routing Protocols for High Vehicular Density VANETs
}

\author{
Álvaro T. Amaya, Alexandre A. P. Pohl, Ricardo Lüders
}

\begin{abstract}
The vehicular Ad-hoc network (VANETs) is a challenging scenario for routing protocols. The high mobility of nodes is responsible for fast changes of topology and frequent interruptions of links. To cope with this challenge, Greedy Perimeter Stateless Routing (GPSR) and Ad-hoc On-demand Distance Vector (AODV) routing protocols have been devised and widely employed in VANETs. However, most approaches have not properly studied the impact of transmission rates and the effect of time intervals of beacons (Hello messages) in the performance of these protocols. In order to address this impact, this work presents an evaluation of GPSR and AODV protocols in an urban scenario of high vehicular density regarding transmission rates and Hello messages intervals. Results show better performance of GPSR for high transmission rate and high traffic density when compared with AODV. Likewise, GPSR achieves more benefits and fewer impacts from Hello messages than AODV.
\end{abstract}

Keywords - Vehicular ad-hoc network, Routing protocol, High traffic density, Beacon, Hello message.

\section{INTRODUCTION}

Vehicular Ad-hoc Networks (VANET) is an emerging technology aimed to provide communication between vehicles (V2V), between vehicles and infrastructure (V2I), between vehicles and persons (V2P), or even vehicle to everything (V2X) [1]. VANETs are considered inheritors of Mobile Adhoc Networks (MANETs), which have benefited from technologies, such as short radio transmission ranges, low bandwidth, self-organization, and self-management [2]. Nonetheless, a VANET presents particular challenges, such as a highly dynamic topology and intermittent network connections.

To cope with the challenges, a large variety of routing protocols has emerged. The GPSR protocol is one of the most typical and promising routing protocols for VANETs [2]. Several works in the literature have shown the advantages of GPSR over other routing protocols in urban scenarios. For instance, Zone Routing Protocol (ZRP) [3], Dynamic Source Routing (DSR) [2], and AODV [2]-[4] have been considered, particularly regarding the average delay. However, most of them have not considered important communication features, such as the transmission rate and the impact of modifying the Hello message transmission interval. Moreover, most researchers employ specific mobility scenarios using a

Álvaro T. Amaya, CPGEI, Federal University of Technology - Paraná (UTFPR), Curitiba-PR, e-mail: amaya@alunos.utfpr.edu.br; Alexandre A.P. Pohl, CPGEI, Federal University of Technology - Paraná (UTFPR), CuritibaPR, e-mail: pohl@utfpr.edu.br; Ricardo Lüders, CPGEI, Federal University of Technology - Paraná (UTFPR), Curitiba-PR, e-mail: luders@utfpr.edu.br fixed number of randomly distributed vehicles, which impose difficulties for comparison with other approaches.

This work seeks to compare the performance of GPSR and AODV routing protocols for VANETs in high vehicular density urban scenarios. For that, we use different transmission rates and time intervals of Hello messages, two features that have not been extensively employed to assess the routing performance. The principal motivation is to explore the harness of Hello messages to support cooperative driving strategies under highly congested conditions. Unlike previous works, our approach explores a wide range of transmission rates as well as a set of different Hello packet time intervals. Furthermore, we employ the vehicular traffic density as an independent parameter to compute the communication performance metrics, and to compare them with similar results found in the literature.

The main contribution of this work is to provide new information about GPSR and AODV protocol's performance in VANETs for high vehicular density scenarios. The novelty consists in assessing the impacts of time intervals of Hello messages and transmission rates, which still has not been addressed to the best of our knowledge.

This paper is organized as follows. Section II provides a description of related works with background material in Section III. Section IV describes the mobility model and the network environment. The results and discussion are presented in Section V, followed by concluding remarks in Section VI.

\section{RELATED WORK}

Most works of the literature focus on the comparison of GPSR with other well-established protocols. For example, the authors of [5] present a taxonomy and evaluation of routing protocols for VANETs. In that work, AODV, DSDV, OLSR, GPSR, and GPCR were studied in an urban area with 20 to 90 vehicles distributed over $2.25 \mathrm{~km}^{2}$, which represents a maximum density of $40 \mathrm{veh} / \mathrm{km}^{2}$. The authors found a constant response from all the main metrics, and the GPSR was considered the best choice based on the average delay metric. However, the packet rate or the Hello time interval is neither informed nor considered for the evaluation.

Another comparison can be found in [6], where the authors present a performance analysis of AODV and GPSR routing protocols. In that work, both urban and highway scenarios were studied. The urban scenario corresponds to an area of $1 \mathrm{~km}^{2}$ and includes two traffic lights. The number of nodes ranges from 15 to 45 , which represents a maximum density 
of $45 \mathrm{veh} / \mathrm{km}^{2}$. In this work, the authors found an almost constant response of GPSR as the number of nodes increases. However, some of their results contradict the findings of [5], particularly for the Packet Delivery Ratio (PDR), and the Average Delay response. Moreover, they do not consider either the transmission rate or the Hello time interval impacts.

Other approaches propose enhanced versions of GPSR, as in [2], where authors present a predictive GPSR named KFGPSR, and an extension called BOD-KF-GPSR for using ondemand Hello messages. In that work, a highway scenario with 50 up to 350 vehicles into an area of about $3.5 \mathrm{~km}^{2}$ were employed. That means a maximum density of $100 \mathrm{veh} / \mathrm{km}^{2}$. The results show that the basic GPSR outperforms AODV, DSR, and ZRP. However, the authors do not comment on the impact of the transmission rates nor the Hello interval used.

Unlike the approaches described above, we explore the performance of both AODV and GPSR protocols considering two parameters that have not been used in previous works: i) the transmission rate; ii) the Hello time interval. To this aim, we employ an urban mobility scenario with higher traffic density than other studies ranging from 62.5 to $312.5 \mathrm{veh} / \mathrm{km}^{2}$.

\section{BACKGROUND}

Routing protocols for VANETs can be classified as topology-based routing (TBR) and position-based routing (PBR) protocols [4]. Furthermore, TBR protocols can be either proactive or reactive. A typical example of a proactive protocol is the Destination-Sequenced Distance Vector (DSDV) protocol, in which a routing table containing information of each node is continuously updated. In reactive protocols, a typical example is the Ad-hoc On-demand Distance Vector (AODV) protocol, which creates routes as long as they are needed.

\section{A. $A O D V$}

AODV is one of the most common routing protocols for mobile ad-hoc networks [7]. AODV combines the use of both destination sequence number and the on-demand route technique. This technique can cause low overhead as the nodes do not have to maintain unnecessary route information.

To handle route information, AODV utilizes three different kinds of route messages: Route Request (RREQ), Route Reply, (RREP), and Route Error (RERR). The route discovery consists of two phases: i) sending of RREQ through the network; ii) looking for a destination and waiting for RREP [7].

Hello packets are locally periodic broadcast messages which are used to indicate link activity. In AODV, Hello messages and broadcast RREQ packets serve as the same function. Therefore, the transmission of RREQ packet indicates the presence of a link. A failure to receive a Hello packet within a time interval indicates a loss of connectivity to that neighbor [7].

\section{B. GPSR}

Different from TBR, PBR protocols do not need to create a routing table or store information of routes. They make the next-hop selection by considering the neighbor position information as well as their position. The Greedy Perimeter
Stateless Routing (GPSR) protocol is a PBR protocol that selects the next-hop for transmission in a greedy manner. If the greedy mode fails, the algorithm switches to the perimeter mode, and the next forward node is selected using the righthand rule [8].

Each node is supposed to know its position information, which is available via GPS or short-range localization, and is expected to periodically exchange that information with its one-hop neighbor through beacon messages (Hello packets). The beacons are periodically transmitted by each vehicle and can include a wide variety of information, such as position, velocity, density, and direction of the vehicles [9].

Based on the information of Hello packets, the source node chooses the node which is closer to the destination. However, if the source does not receive any response from a neighbor within a time-out interval, it considers the communication link as broken. There may be a situation where the source does not find a better neighbor than itself. This situation is known as the local-maximum condition in which GPSR can no longer follow the greedy forwarding strategy. In this case, the protocol switches to the perimeter mode [9].

\section{Simulation CONFIGURATION}

A typical urban mobility scenario has been chosen in order to study the performance of AODV and GPSR protocols in a VANET. It corresponds to a squared region of $0.16 \mathrm{~km}^{2}$ including a two-road crossing scenario with a traffic light. Each road has three one-way lanes as shown in Fig. 1.

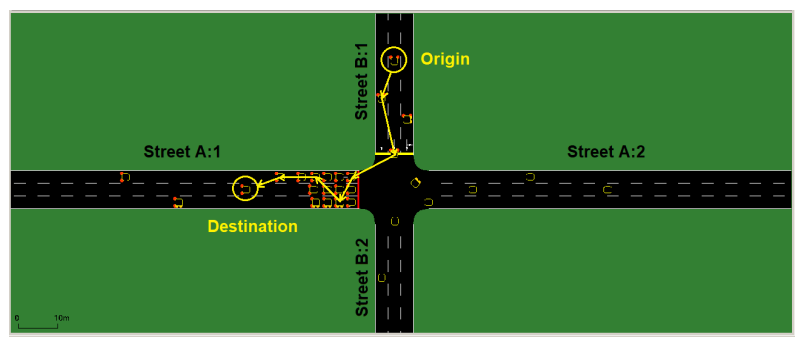

Fig. 1. Two-road crossing scenario.

Each road of Fig. 1 is composed of two streets: A:1 and A:2 for route $\mathrm{A}$, and $\mathrm{B}: 1$ and $\mathrm{B}: 2$ for route $\mathrm{B}$. The traffic flows are such that vehicles on $\mathrm{A}: 1$ can pass through the crossing section and go through $\mathrm{A}: 2$, or turn right to take $\mathrm{B}: 2$. Likewise, the vehicles coming from $\mathrm{B}: 1$ can go through $\mathrm{B}: 2$ or $\mathrm{A}: 2$.

As for the communication scheme, a vehicle in route $\mathrm{B}$ (origin) tries to communicate with a vehicle in route A (destination). The origin vehicle goes through streets $\mathrm{B}: 1$ and $\mathrm{B}: 2$, and the destination vehicle through $\mathrm{A}: 1$ and $\mathrm{A}: 2$. Both vehicles stop at the traffic light in such a way that the destination vehicle is the first one to pass through the intersection.

According to the mobility scenario, the communication scheme involves three phases: i) the phase before both vehicles achieve the intersection when the communication depends on relaying hops; ii) the phase when the origin and destination vehicles meet each one at the intersection, establishing a direct communication; iii) the phase after the destination vehicle 
passes through the intersection when the communication will depend on relaying hops again.

The transmission starts as soon as both vehicles enter the area at $25 \mathrm{~s}$ of simulation time, and ends when one of them leaves the area at time $65 \mathrm{~s}$.

Mobility patterns are created with the SUMO simulator [10] for $10,20,30,40$, and 50 vehicles, which result in traffic densities of $62.5,125,187.5,250$, and $312.5 \mathrm{veh} / \mathrm{km}^{2}$, respectively. For each case, the traffic density is carefully adapted to keep a uniform distribution of cars for each simulation.

The IEEE 802.11p standard with AODV and GPSR protocols are employed for the simulation of the network using NS2 [11]. For both protocols, a CBR/UDP data traffic of different transmission rates and time intervals of Hello messages are considered. Other communication parameters are listed in Table I.

TABLE I

COMMUNICATION PARAMETERS.

\begin{tabular}{|l||l|}
\hline Parameter & Value \\
\hline \hline Region dimension $\left(\mathrm{m}^{2}\right)$ & $400 \times 400$ \\
\hline Traffic density $\left(\mathrm{veh} / \mathrm{km}^{2}\right)$ & $62.5,125,187.5,250,312.5$ \\
\hline Mobility model & Traffic lights \\
\hline Lane configuration & Two flows one way \\
\hline Maximum speed & $50 \mathrm{~m} / \mathrm{s}$ \\
\hline Driving model & Krauss \\
\hline Channel type & Wireless \\
\hline Simulation time & $45 \mathrm{~s}$ \\
\hline Traffic type & CBR/UDP \\
\hline Propagation model & TwoRayGround \\
\hline MAC/PHY & IEEE 802.11p \\
\hline Radio range & $150 \mathrm{~m}$ \\
\hline Packet size & 512 bytes \\
\hline Transmission rate (Mbps) & $0.5,1,3,6,12$ \\
\hline Routing protocols & AODV, GPSR \\
\hline
\end{tabular}

The results are evaluated according to the following metrics. Packet Delivery Ratio (PDR): the ratio between the amount of received and sent packets [3].

$$
P D R=\frac{\text { Total number of received packets }}{\text { Total number of sent packets }}
$$

End-to-End Delay (E2E Delay): the average time interval taken to transmit a packet from a source to a destination [3].

$$
E 2 E \text { Delay }=\frac{\sum(\text { Packet arrival time }- \text { Packet departure time })}{\text { Total number of received packets }}
$$

Throughput: the total amount of received bits per time unit delivered from one node to another [3].

Throughput $=\frac{\text { Total number of received packets } \times \text { Packet size }}{\text { Total simulation time }}$

These performance metrics are computed as average values for different traffic densities. They capture the impact of traffic density in the communication under different transmission rates and Hello intervals, even considering that some dispersion is expected.

\section{RESULTS AND DISCUSSION}

This section presents the performance of AODV and GPSR protocols regarding PDR, End-to-End Delay, and Throughput for different transmission rates, and time intervals of Hello messages by considering different densities of vehicles.

\section{A. Impact of the transmission rate}

The results of packet delivery ratio (PDR) per vehicular traffic density for transmission rates of $0.5,1,2,3,6$, and $12 \mathrm{Mbps}$ are shown in Fig. 2. Results show that PDR decreases as the transmission rate increases for both AODV and GPSR scenarios. Differences between AODV and GPSR are more representative at low transmission rates and high-density scenarios. That occurs because the routing table of AODV is more advantageous when multiple routing choices are available, and stable links with low transmission rates entail fewer drops of packets.

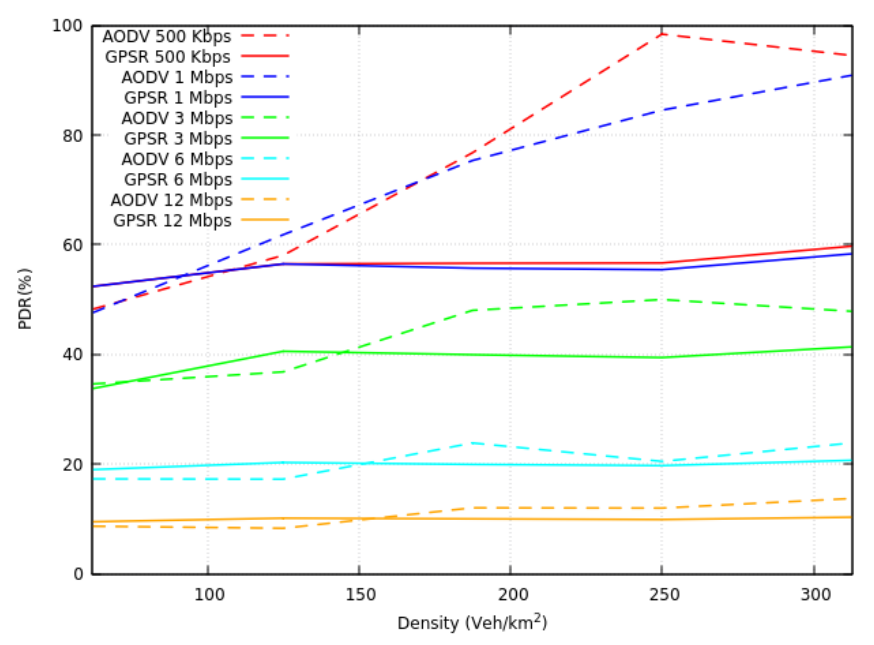

Fig. 2. Packet delivery ratio (PDR) per vehicular traffic density for AODV and GPSR using different transmission rates.

The constant behavior of PDR in GPSR is consistent with the findings of [5], [12], [2], and [6], but somewhat different from [8] whose authors argue that PDR increases with density for GPSR. Nevertheless, we found that the PDR using AODV does not decrease as density increases according to [5].

Another important discussion is whether PDR is higher for GPSR than for AODV. For instance, [5] claim that PDR for AODV is higher than for GPSR, but [6] claim the opposite. We found that more prominent differences become evident for very high densities and low transmission rates. In these scenarios, AODV outperforms GPSR.

The results of End-to-End Delay (E2E Delay) per traffic density for transmission rates of $0.5,1,2,3,6$, and $12 \mathrm{Mbps}$ are shown in Fig. 3. The most evident outcome is the big difference between GPSR and AODV results. In general, the E2E delay for AODV is higher than for GPSR. Additionally, an incremental trend is realized with AODV, whereas a constant E2E Delay is observed with GPSR. We observe that the greedy feature of GPSR leads to deliver the packets through shorter paths than in AODV, thus reducing the average end-to-end delay.

Other particular behaviors can be seen in Fig. 3. For instance, the E2E Delay at transmission rates lower than $3 \mathrm{Mbps}$ is quite different from those above that value. For values above 3 Mbps, AODV shows a high and similar response for low densities, whereas GPSR shows a reduction of E2E Delay as 
of $3 \mathrm{Mbps}$ onwards. For transmission rates under $3 \mathrm{Mbps}$, the results of each protocol are lower than in the other three rates.

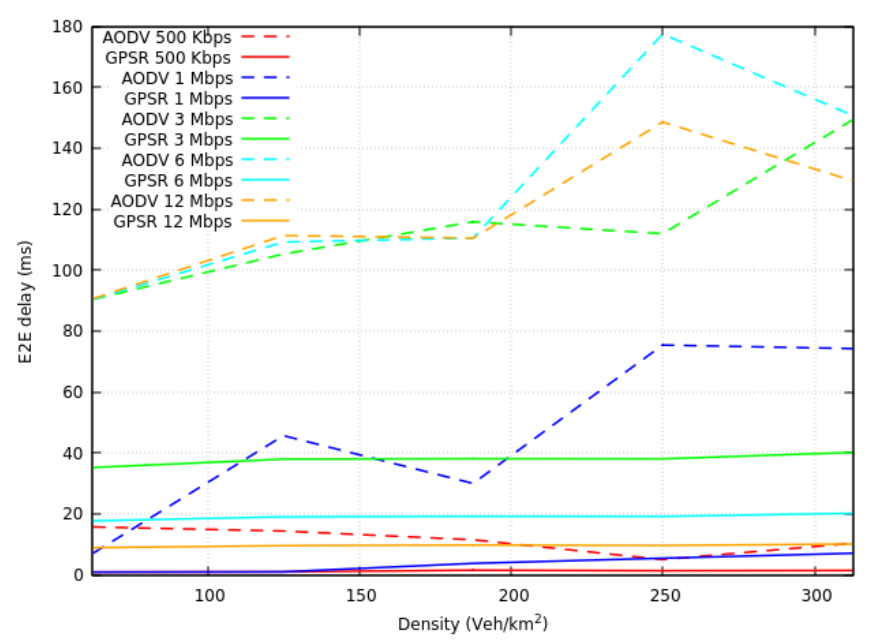

Fig. 3. End-to-End Delay per vehicular traffic density for AODV and GPSR using different transmission rates.

The remarkable difference concerning delay for AODV and GPSR is consistent with the findings of [5], [12], [2], and [8], but it is different of [6] whose authors found that the E2E-Delay is negligible and equal for both protocols, which contradicts [5] for a similar traffic density.

The results of throughput per traffic density for transmission rates of $0.5,1,2,3,6$, and 12 Mbps are shown in Fig. 4. It shows that, in general, the throughput increases as the transmission rate increases, achieving a maximum of around 1.5 Mbps. Fig. 4 also shows that, differently from AODV, the results for GPSR are more stable as density increases. It also shows that for transmission rates higher than $3 \mathrm{Mbps}$, GPSR outperforms the AODV.

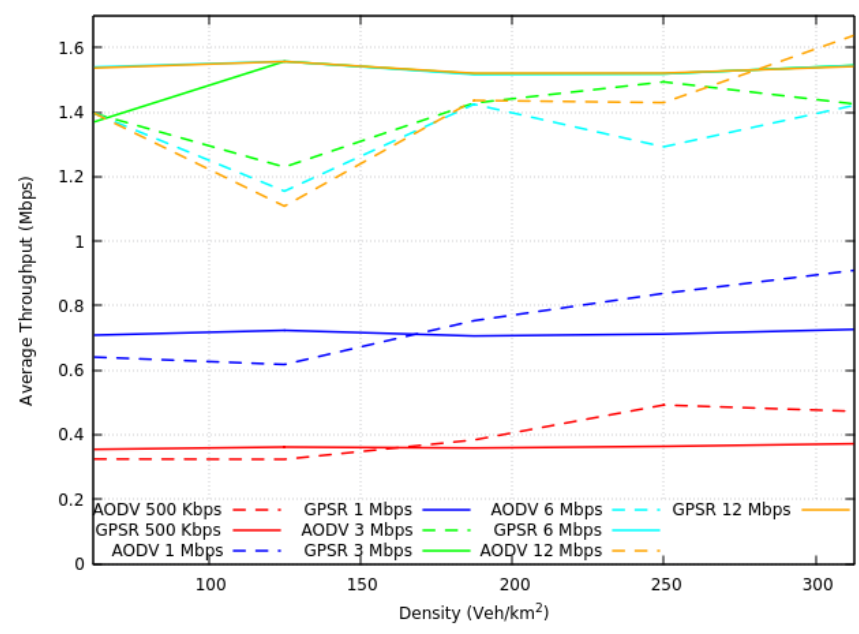

Fig. 4. Throughput per vehicular traffic density for AODV and GPSR using different transmission rates.

The almost constant throughput of GPSR for different vehicular densities in Fig. 4 is consistent with the results of [5]. However, the authors of [5] claim that the throughput in AODV is higher than in GPSR when considering a maximum density of $13 \mathrm{veh} / \mathrm{km}^{2}$. We found this is true only for high-density scenarios. For low densities (under $100 \mathrm{veh} / \mathrm{km}^{2}$ ) GPSR shows a slightly higher throughput than AODV as evidenced in [2] for densities between 14.28 and $100 \mathrm{veh} / \mathrm{km}^{2}$.

\section{B. Impact of the time interval between Hello messages}

As mentioned before, the routing protocol can be aware of mobility changes in more quick and accurate localization of hops by using Hello packets. However, a channel overhead is expected to occur as Hello packets are transmitted along with other communication traffic. In this section, an analysis of the performance of both protocols is described. We employ a transmission rate of $3 \mathrm{Mbps}$, which has shown a regular behavior for using in VANETs. .

The same three communication metrics (i.e. PDR, E2E Delay, and Throughput) applied to CBR/UDP traffic are employed again. The results of packet delivery ratio (PDR) per vehicular traffic density for different Hello time intervals are shown in Fig. 5. Different from AODV, where RREQ can perform the Hello function, GPSR depends on Hello messages, so no curve for No Hello was considered in GPSR. The results show that GPSR is more stable than AODV, and it seems to converge to a constant value as the Hello time interval decreases.

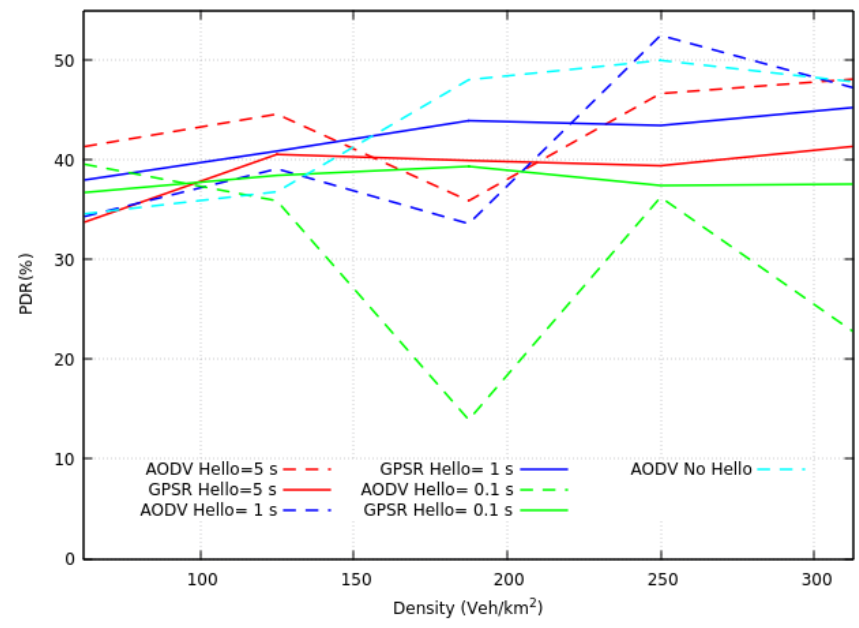

Fig. 5. Packet Delivery Ratio (PDR) per vehicular traffic density for AODV and GPSR using different Hello time intervals.

The results of End-to-End Delay (E2E Delay) per traffic density for different Hello time intervals are shown in Fig. 6. The results show that the reduction of the time of Hello interval causes more impacts in AODV than in GPSR. Furthermore, for low densities, no increments of E2E Delay are obtained with GPSR. As evidenced in PDR, stability remains to be a characteristic feature of GPSR in the E2E Delay.

Also from Fig. 6 a particular out of fit point for AODV at $187.5\left(\mathrm{veh} / \mathrm{km}^{2}\right)$ becomes evident. It is a singular case where the protocol can not keep a stable path between origin and destination nodes. There exist two possible behaviors. First, to rule out a large number of packets, as evidenced in Fig. 5 


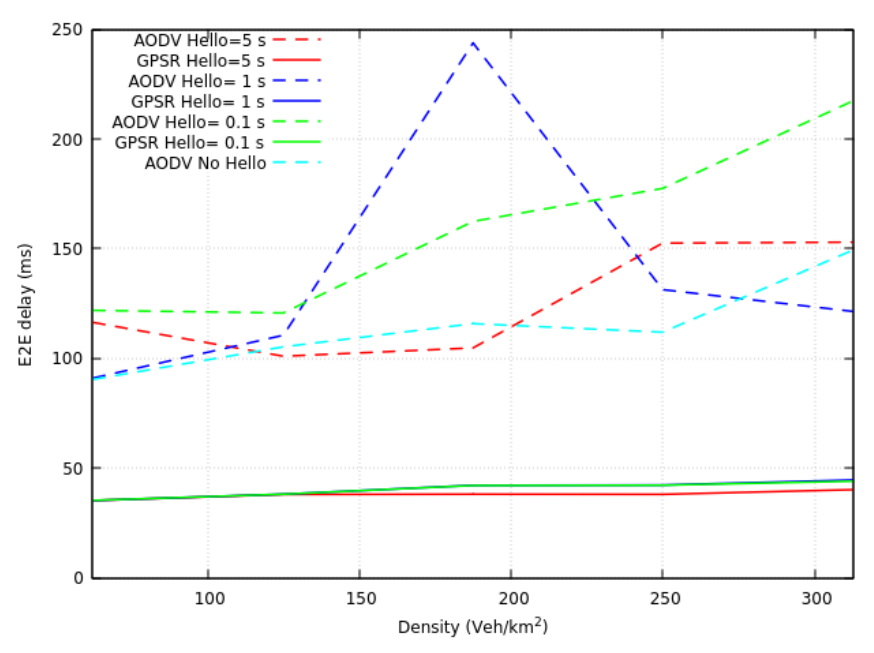

Fig. 6. End-to-End Delay per vehicular traffic density for AODV and GPSR using different Hello time intervals.

for the Hello interval of 0.1 seconds, and second, to persist in the transmission of the packets, despite the high time cost, as in the case of Hello interval of 1 second in Fig. 6 (blue dashed curve). The Fig. 6 let to see that GPSR performs better in those situations.

The results of throughput per vehicular traffic density for different Hello time intervals are shown in Fig. 7. We see that the impact of reducing the Hello time interval in very high densities is to reduce the throughput, particularly for the AODV case. At lower densities (under $100 \mathrm{veh} / \mathrm{km}^{2}$ ), the effect of Hello messages is to improve the throughput. A particular case is the use of a Hello interval of $1 \mathrm{~s}$ in GPSR. In this case, the throughput is higher than in other GPSR cases for any density.

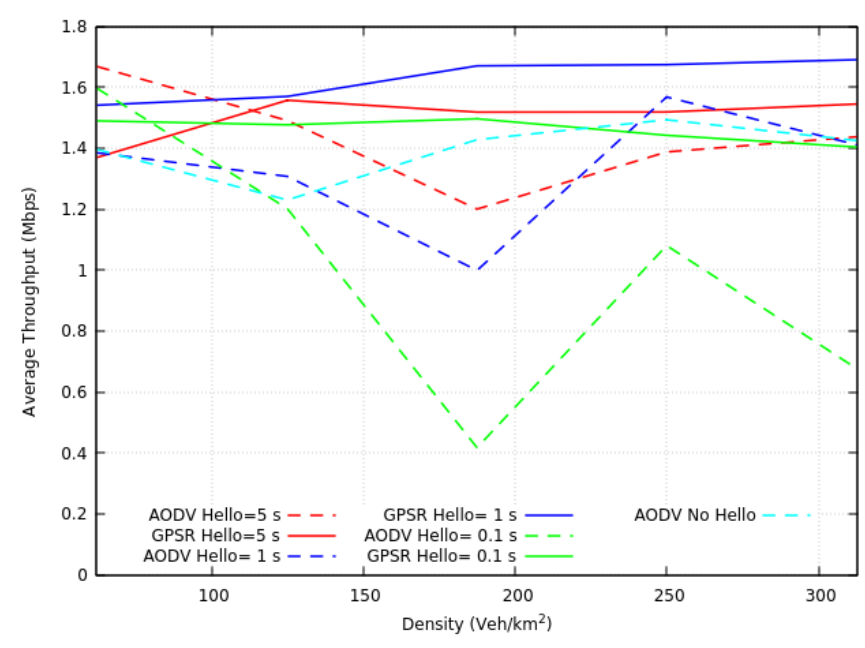

Fig. 7. Throughput per vehicular traffic density for AODV and GPSR using different Hello time intervals.

\section{CONCLUSION}

In this work, three communication metrics to assess the performance of AODV and GPSR protocols in VANETs con- sidering high vehicular traffic densities have been employed: i) Packet Delivery Ratio; ii) End-to-End delay; iii) Throughput. GPSR appears to be the best choice for high density and high transmission rate scenarios, mainly because of the End-to-End Delay and Throughput results, which are better than in AODV. Although PDR results of GPSR do not outperform those of AODV, particularly for high density of vehicles, GPSR is less sensitive to variations of vehicular traffic density than AODV.

Furthermore, this work has shown the effect of using Hello packets with the GPSR and AODV protocols in VANETs. Results show that the GPSR achieves more benefits and less negative impacts from Hello packets when the interval is modified. For very high densities, a time interval as short as $100 \mathrm{~ms}$ worsens all metrics, particularly for the AODV protocol. However, a time interval of one second contributes to better performance of GPSR, although it is not beneficial for AODV.

Future works will address the performance of the GPSR protocol when used in the transmission of timely-critical kinematic data for connected vehicles.

\section{ACKNOWLEDGMENT}

This study was financed in part by the Coordenação de Aperfeiçoamento de Pessoal de Nível Superior-Brasil (CAPES)-PEC-PG.

\section{REFERENCES}

[1] Y. Allouche and M. Segal, "Cluster-based beaconing process for VANET," Vehicular Communications, vol. 2, no. 2, pp. 80-94, apr 2015.

[2] Z. Houssain, I. Zaimi, M. Drissi, M. Oumsis, and S. E. A. Ouatik, "Trade-off between accuracy, cost and QoS using beacon on demand strategy and Kalman filtering over vanet, digital communications and networks," 2017.

[3] A. Setiabudi, A. A. Pratiwi, D. Perdana, R. F. Sari et al., "Performance comparison of GPSR and ZRP routing protocols in vanet environment," in 2016 IEEE region 10 symposium (TENSYMP). IEEE, 2016, pp. $42-47$.

[4] L. Hu, Z. Ding, and H. Shi, "An improved GPSR routing strategy in VANET," in 2012 8th International Conference on Wireless Communications, Networking and Mobile Computing. IEEE, 2012, pp. 1-4.

[5] A. Bengag, A. Bengag, and M. Elboukhari, "Routing protocols for VANETs: A taxonomy, evaluation and analysis," Advances in Science, Technology and Engineering Systems Journal, vol. 5, no. 1, pp. 77-85, 2020.

[6] R. Bala and C. R. Krishna, "Scenario based performance analysis of AODV and GPSR routing protocols in a VANET," in 2015 IEEE International Conference on Computational Intelligence \& Communication Technology. IEEE, 2015, pp. 432-437.

[7] H.-P. Wang and L. Cui, "An enhanced AODV for mobile ad hoc network," in 2008 International Conference on Machine Learning and Cybernetics, vol. 2. IEEE, 2008, pp. 1135-1140.

[8] S. Rahimi and M. A. J. Jamali, "A hybrid geographic-DTN routing protocol based on fuzzy logic in vehicular ad hoc networks," Peer-toPeer Networking and Applications, vol. 12, no. 1, pp. 88-101, 2019.

[9] A. Silva, K. Reza, A. Oliveira, and A. Klautau, "A reduced beacon routing protocol for inter-vehicle communications." XXXVII Simpósio Brasileiro de Telecomunicações e Processamento de Sinais, 012019.

[10] M. Behrisch, L. Bieker, J. Erdmann, and D. Krajzewicz, "Sumosimulation of urban mobility: an overview," in Proceedings of SIMUL 2011, The Third International Conference on Advances in System Simulation. ThinkMind, 2011.

[11] T. Issariyakul and E. Hossain, "Introduction to network simulator 2 (ns2)," in Introduction to network simulator NS2. Springer, 2009, pp. $1-18$.

[12] R. Karimi and S. Shokrollahi, "Pgrp: Predictive geographic routing protocol for vanets," Computer Networks, vol. 141, pp. 67-81, 2018. 